\title{
Diálogo interreligioso como clave para la formación ciudadana de estudiantes universitarios ${ }^{1}$
}

Interreligious dialog to the citizens behaviour of univesitaries students

Pablo Palet A.

ppalet@uct.cl

Rommel Sanhueza G.

sg.rommel@gmail.com

Universidad Católica de Temuco

Chile

\section{Resumen}

En el contexto nacional, La Araucanía es una región caracterizada por la diversidad étnica, cultural y religiosa. Al mismo tiempo, la creciente emergencia de la conciencia de identidad y pertenencia al pueblo mapuche y la asimetría de condiciones de vida en relación con la sociedad dominante, han hecho del conflicto entre el Estado de Chile y el pueblo Mapuche un tema de primera relevancia regional.

Por otra parte, la Universidad Católica de Temuco, desde su fundación el año 1959, ha declarado un claro y decidido compromiso con la región buscando comprender y contribuir a la solución de sus problemáticas, desafíos y aspiraciones. La propuesta de esta presentación es que, para cumplir su compromiso público de aportar a la convivencia social, la UC Temuco debe atender a la formación integral de sus estudiantes como profesionales y ciudadanos, una de cuyas dimensiones es la cosmovisión religiosa, lo que

${ }^{1}$ Versión escrita de la ponencia presentada en el II Congreso Nacional sobre el Fenómeno Religioso en el Mundo Contemporáneo, Grupo de Trabajo $\mathrm{N}^{\circ} 10$ Tradiciones religiosas en diálogo, Santiago, Universidad de Chile, 26 de octubre de 2017. 
implica encontrar y defender el estatuto propio de la 'educación de la fe' en el contexto universitario, caracterizado por la 'neutralidad religiosa'.

En este contexto, se propone considerar las condiciones para un auténtico diálogo interreligioso como la perspectiva más adecuada para favorecer el desarrollo de la fe en el ámbito universitario. En este marco se presenta el caso del curso electivo Manifestaciones de la espiritualidad en la Araucanía. Una mirada a la diversidad. Se describen cuatro momentos relevantes de esta experiencia: identidad religiosa, religiones ante la realidad contemporánea, testimonios de fe, y salir de sí al encuentro del otro. Finalmente se presentan algunos resultados significativos para el propósito de aportar a la formación de ciudadanos comprometidos con la convivencia armónica y la paz.

Palabras claves: La Araucanía, espiritualidad mapuche, conflicto mapuche-estado, diversidad, compromiso ciudadano.

\begin{abstract}
In the national context, La Araucanía is a region characterized by ethnic, cultural and religious diversity. At the same time, the growing emergence of awareness of identity and belonging to the Mapuche people and the asymmetry of living conditions in relation to the dominant society have made the conflict between the State of Chile and the Mapuche people a topic of first regional relevance.

On the other hand, the Catholic University of Temuco, since its foundation in 1959, has declared a clear and determined commitment to the region, seeking to understand and contribute to the solution of its problems, challenges and aspirations. The proposal of this presentation is that, in order to fulfill its public commitment to contribute to social coexistence, UC Temuco must attend to the integral formation of its students as professionals and citizens, one of whose dimensions is the religious worldview, which
\end{abstract}


implies finding and defend the proper status of the 'education of the faith' in the university context, characterized by 'religious neutrality'.

In this context, it is proposed to consider the conditions for an authentic interreligious dialogue as the most appropriate perspective to favor the development of the faith in the university environment. In this framework, the case of the elective course Manifestations of spirituality in Araucanía is presented. A look at diversity. Four relevant moments of this experience are described: religious identity, religions before contemporary reality, testimonies of faith, and leaving oneself to meet the other. Finally, some significant results are presented for the purpose of contributing to the formation of citizens committed to harmonious coexistence and peace.

Keywords: Araucania, Mapuche spirituality, conflict between mapuche and the state conflict, diversity, citizen commitment.

\section{A modo de contexto: La Araucanía y la Universidad Católica de Temuco}

Actualmente, la Araucanía se distingue, entre otras cosas, por ser región que pluri-étnica, multicultural e interreligiosa; pero también por la persistencia de un evidente conflicto entre el Estado de Chile y el Pueblo mapuche.

Desde el punto de vista de los datos, lo único que por ahora sabemos del censo 2017 es el total de habitantes por región, que otorga a La Araucanía una población estimada de 938.626 personas, el quinto lugar entre las quince regiones del país. La afiliación religiosa y a pueblos indígenas en cambio, son datos del fallido censo 2012. En este, el 59\% de la población regional se declaró católico (8\% menos que el promedio nacional); 27,5\% evangélicos o protestantes (casi un 11\% por encima de la media nacional), 9,2\% declaró no tener ninguna religión en absoluto, y sólo $0,8 \%$ de la religión indígena (lo que aun así significa casi el $40 \%$ de quienes declararon esta opción en todo el país). Además, la 
presencia mapuche alcanzó el 31,6\% de la población regional, casi tres veces superior a la media nacional de habitantes de pueblos indígenas. Estas cifras permiten sostener que, en el contexto nacional, La Araucanía es una región multi-religiosa, con un peculiar carácter pluri-étnico y multicultural.

Por otra parte, la historiografía contemporánea marca el año 1997 como un hito en el actual conflicto entre el Estado de Chile y el pueblo mapuche, a raíz de la primera quema de tres camiones forestales por parte de la Coordinadora de Comunidades en conflicto Arauco Malleco. ${ }^{2}$ Desde entonces, el uso de la violencia política se ha venido incrementando, con pérdidas materiales de consideración, la militarización de algunas zonas, el desconocimiento por parte de los sucesivos gobiernos del tratamiento político del conflicto, la judicialización de la demanda mapuche, autoridades tradicionales detenidas, niños y mujeres heridos $\mathrm{y}$, lo que es irreparable, la muerte de algunos comuneros, colonos y carabineros.

La Araucanía es una región pluri-étnica, en la que la presencia occidental se impuso como dominación hace 140 años. La asimetría cultural es evidente y los últimos 20 años muestran una sostenida emergencia de la identidad mapuche. ¿Qué rol juega en ello la Iglesia Católica y en particular la Universidad Católica de Temuco (en adelante UC Temuco)?

Desde su fundación el año 1959, esta Universidad se ha caracterizado por su compromiso público con la región de La Araucanía. Es parte esencial de su misión en cuanto Universidad y en cuanto Católica. A modo de ejemplo: se instalan las Semanas Indigenistas en 1963, más tarde se crea la carrera de Antropología a principios de los 70, y de Pedagogía Básica intercultural en 1992. En los últimos años se funda la Cátedra Fray Bartolomé de Las Casas en 2009. Todo ello son ejemplos de la vocación pública y regional de la Universidad, con particular foco en la situación del pueblo mapuche.

En la actualidad, el Plan de Desarrollo Institucional declara que la

${ }^{2}$ Fernando Pairican, Malon. La rebelión del movimiento mapuche 1990-2013, Santiago: Pehuén, 2016. 
Universidad Católica de Temuco ha sido fundada por la Iglesia Católica, en el espíritu del

Evangelio de Jesucristo y el Magisterio de la Iglesia, para servir a la sociedad de La Araucanía y la macro región sur del país, dedicándose desde sus inicios a "la formación amplísima de los futuros profesionales, al cultivo de las ideas que abren nuevos surcos al saber, y a la investigación que perfecciona la vida y las instituciones, generando nuevos horizontes y posibilidades a su gente y sus territorios". 3

Para lograrlo se "vincula con su entorno social para comprender y contribuir a la solución de sus problemáticas, desafíos y aspiraciones, inspirada en la Doctrina Social de la Iglesia"4 ¿ ¿Cómo se realiza esto en una región marcada, como hemos bosquejado, por la interculturalidad, inter-etnicidad y el conflicto político social vinculado?

\section{Formación integral y educación de la fe en el contexto universitario}

Para cumplir adecuadamente su compromiso público de aportar a la convivencia ciudadana, en un contexto social de diversidad étnica, cultural y religiosa, la UC Temuco debe atender con particular énfasis la formación personal de sus estudiantes, la formación a la sabiduría en palabras del papa Francisco ${ }^{5}$, una de cuyas dimensiones insoslayables es la cosmovisión religiosa. Y, ¿por qué es insoslayable la dimensión religiosa?

${ }^{3}$ Alejandro Menchaca Lira, Decreto $N^{\circ} 375$ Diócesis San José de Temuco, 8 de septiembre de 1959.

${ }^{4}$ Universidad Católica de Temuco, Plan de Desarrollo Institucional 2010-2020, Temuco, 2010.

5 Papa Francisco, Discurso al mundo universitario en la Pontificia Facultad Teológica Regional en Cerdeña (22 de septiembre de 2013); disponible en https://w2.vatican.va/content/francesco/es/speeches/2013/september/ documents/papafrancesco_20130922_cultura-cagliari.html 
En primer lugar porque la religión es el corazón de la cultura, y entonces subyace en nuestras concepciones de hombre y mujer, de sociedad, de organización política y económica, etc. Si pretendemos formar profesionales integrales no centrados en su propio beneficio y estatus, sino con capacidad de construcción crítica de la sociedad y de convivencia ciudadana, profesionales con visión respecto de su propio rol en la construcción de cultura cívica, entonces hay que asumir la cosmovisión religiosa.

Pero además, en el contexto intercultural de La Araucanía reconocemos que en las religiones tradicionales no hay separación entre religión y vida. En la mentalidad indígena la religión no es una idea a creer o un conjunto de acciones a practicar; no es algo que yo elijo, sino algo a lo que pertenezco, y que incluye la comunidad y el territorio. En el caso del pueblo mapuche, su identidad tiene que ver con una forma de relacionarse y comprender el тари (la tierra) en su dimensión material e inmaterial. Мapu es el lugar que habita el ser humano y que le da sustento, el espacio físico; la misma palabra designa al territorio comunitario en su dimensión sociopolítica, el país del pueblo mapuche; y también se usa mapu para designar la cosmovisión que permite comprender el universo en sus ocho dimensiones espaciales. Entonces, una formación ciudadana que asuma la perspectiva de diálogo intercultural con el pueblo mapuche para contribuir así a una gestión armónica del conflicto, no puede sino asumir la dimensión religiosa involucrada en toda cultura. Por eso es necesario que la Universidad tenga un rol en la "educación de la fe".

Sin embargo, ¿cómo "educar la fe" en el contexto universitario y regional? Esto parece comportar un doble desafío:

- La educación superior occidental parece forzar la neutralidad religiosa en las universidades, o por lo menos que la fe religiosa debe quedar entre paréntesis para evitar la contaminación de la verdad pública y objetiva. Se instala la idea que con recursos públicos no corresponde hacer proselitismo ni catequesis; y efectivamente en una universidad, por su carácter de apertura al conocimiento y búsqueda de la verdad, no puede exigirse una confesión determinada como requisito para estudiar o enseñar. 
- Lo anterior impulsa a un cambio de paradigmas, desde aquel de la transmisión de un mensaje religioso hacia uno de adquisición de competencia religiosa. Esto es, que los estudiantes desarrollen la capacidad para percibir a través de los elementos de la religión la experiencia de lo sagrado que tiene el sujeto religioso, así como el sentido del mundo y de la vida que la religión le proporciona, y de responder a su estímulo de forma adecuada a las propias opciones. ${ }^{6}$ Así, se intenta ser coherentes con tres ejes del modelo educativo de la UC Temuco: basado en competencias, centrado en el aprendizaje significativo de los estudiantes, con inspiración humanista cristiana.

En particular, el enfoque de formación humanista cristiana que inspira el conjunto de la función docente en la UC Temuco implica entender la educación como una actividad humana natural, holística, histórica y política que involucra tanto a la sociedad y el individuo. Si bien las dos primeras ideas enfatizan el proceso individual de llegar a ser personas humanas completas, los dos últimos conceptos subrayan los aspectos sociales y comunitarios del proceso educativo. Sin embargo, ninguno de ellos puede ser bien entendido separado de los demás. Si la educación es la tarea de llevar a cabo todas las potencialidades del ser humano, desarrollado por él mismo y su comunidad, tiene un objetivo trascendente inherente.

Por otra parte, este enfoque también implica re contextualizar la comprensión de la fe religiosa, sin identificarla con un asentimiento cognitivo a ciertas doctrinas, ni con la sensación emotiva de estar ligado a Dios, menos con la mera adhesión a un código de conducta ética. Sin descartarlos, la perspectiva es más amplia. La fe es "reconocida como la actividad de buscar y descubrir el significado de las dimensiones más amplias de nuestra experiencia."7 Siguiendo a James Fowler, ${ }^{8}$ aquí se entiende la fe como una iniciativa relacional de una persona dentro de su comunidad (es decir, sus otros

${ }^{6}$ Rafael Artacho López, Enseñar competencias sobre la religión: Hacia un currículo de Religión por competencias, Bilbao: DDB 2009; p. 40 y 86.

7 Sharon Parks, Big Questions, Worthy Dreams: mentoring Young adults in their search for meaning, purpose, and faith, San Francisco: Jossey-Bass Inc., 2000; p. 7. Traducción propia.

8 James Fowler, Stages of Faith. The Psychology of Human Development and the Quest for Meaning, San Francisco: Harper \& Row, 1976.

Cuadernos Judaicos ISSN: 0718-8749 
significativos) y su centro compartido de valor y poder. La fe es un acto de la personalidad en su conjunto, que participa en la dinámica de la vida personal y social vivida de acuerdo a los valores últimos. Por mucho que se vincule a la conciencia personal, la fe también es comunitaria en la medida en que se expresa en símbolos y mitos que no pueden ser plenamente entendidos fuera de la comunidad. La razón, entendida como la estructura significativa de la mente y de la realidad y no sólo en su sentido científico-técnico, hace la fe comprensible y comunicable, lo que permite compartir su acceso a la verdad más amplia, sin negar la duda (elemento intrínseco a la fe en esta comprensión).

Nos parece que esta comprensión amplia de la fe, como dimensión antropológica fundamental del ser humano, así como de educación en su sentido holístico y político, hacen posible hablar de "educación de la fe" en el contexto universitario, por naturaleza plural y diverso.

\section{El diálogo interreligioso como clave}

El diálogo interreligioso lo entendemos como el encuentro y la escucha mutua entre personas de diferentes cosmovisiones; es un diálogo sobre cuestiones de fe o religión sustentado en las convicciones personales profundas que movilizan existencialmente a cada persona. Busca contribuir al crecimiento personal y al beneficio de la sociedad, sobre todo en la búsqueda del bien común y de la paz. Por lo tanto, parte de la base que quienes dialogan son personas interesadas en crecer en su fe y religiosidad, entendida en el sentido amplio de "espiritualidad «humana fundamental», ético-política" al decir de Casaldáliga, 9 por lo que el diálogo interreligioso no es un intento de conversión ni menos una instancia de proselitismo.

Actualmente el diálogo interreligioso es una situación nueva a la cual se ven enfrentadas las personas y muchas prefieren dejar esta responsabilidad en manos de las autoridades

9 Pedro Casaldáliga y José María Vigil, Espiritualidad de la Liberación, Santander: Sal Terrae, 1992; p. 19.

Cuadernos Judaicos ISSN: 0718-8749 
eclesiásticas o de los estudiosos de las religiones. Ante esto y respondiendo a la invitación del Concilio Vaticano II parece necesario que todos los creyentes -manteniendo su feentren en diálogo con los fieles de otras religiones, e incluso con el creciente número de personas que se declaran 'sin religión'.

Por consiguiente, exhorta a sus hijos a que, con prudencia y caridad, mediante el diálogo y colaboración con los adeptos de otras religiones, dando testimonio de fe y vida cristiana, reconozcan, guarden y promuevan aquellos bienes espirituales y morales, así como los valores socio-culturales que en ellos existen. ${ }^{10}$

Por último hay que considerar que el diálogo interreligioso, en cuanto necesidad de la sociedad contemporánea, requiere que los interlocutores dejen de lado su pretensión de superioridad respecto de otras religiones. Este diálogo sólo es eficaz si se considera que todas las religiones tienen algo que aportar al bien común y es posible en la medida en que cada uno se comprometa de manera efectiva a llevar adelante un proyecto común que mejore las relaciones, promueva la paz y la armonía conducentes a una sana convivencia. Se trata entonces de generar espacios, una instancia de vida compartida, que busca contribuir a una mejor sociedad, con el aporte de lo mejor de cada uno.

En el contexto de la adscripción religiosa en La Araucanía, mencionada al inicio, pareciera más pertinente referirse al ecumenismo, esto es, al diálogo entre cristianos de diferentes Iglesias y denominaciones, que tiene como objetivo final la unidad de los cristianos. Para los efectos de la experiencia formativa que sigue, se lo entiende como tal y diferente del

${ }^{10}$ Concilio Vaticano II, Nostra Aetate, Proemio 2.

Cuadernos Judaicos ISSN: 0718-8749 
diálogo interreligioso; ${ }^{11}$ sin embargo, se ha incorporado a los participantes de diferentes iglesias cristianas como partes del diálogo interreligioso.

\section{Experiencia de diálogo interreligioso: el curso Manifestaciones de la Espiritualidad}

En el trascurso de estos 58 años, la Universidad Católica de Temuco ha generado nuevos escenarios formativos para responder de manera adecuada a los desafíos de la sociedad. Es así como en el año 2005, se crearon los cursos Electivos Interdisciplinarios, que hoy conforman el Programa de Formación para la diversidad en los que se desarrolla y refuerza, entre otras, la competencia genérica Respeto y Valoración de la diversidad, definida como:

Se reconoce y reconoce a los demás en sus diferencias humanas, sean éstas socioeconómicas, etnoculturales, político-ideológicas, religioso-espirituales, etarias, de género, afectivo-sexuales, de capacidades fisiocognitivas, u otras, y favorece un diálogo intercultural humanizador y promotor de entornos sociales más justos e inclusivos. $^{12}$

Uno de los cursos electivos que forma parte de este programa es el llamado Manifestaciones de la espiritualidad en la Araucanía. Una mirada a la diversidad. Tiene como objetivos que los estudiantes reconozcan su dimensión espiritual y religiosa, descubran lo que los diferencia a unos de otros y visualicen intereses comunes que puedan guiarlos a actos cooperativos, fundamentalmente la búsqueda de la paz, en los distintitos ámbitos del quehacer personal, profesional y social. Esto es esencial puesto que responde a

${ }^{11}$ Juan Pablo García Maestro, "Retos del Diálogo Interreligioso al Diálogo Ecuménico" en Cultura para la Esperanza 63 (2006), p. 31-33; disponible en http://www.accionculturalcristiana.org/html/revista/r63/63dial.pdf

12 Dirección General de Docencia UCTemuco, Competencias genéricas para la formación de profesionales integrales, Temuco: Ediciones UC Temuco, 2016.

Cuadernos Judaicos ISSN: 0718-8749 
los desafíos de vivir y trabajar en una región, como se ha dicho, marcada por el conflicto entre el Estado chileno y el Pueblo Mapuche, y la necesidad de encontrar formas de convivencia social interculturales e interreligiosas. Para lograr estos objetivos, el curso invita a los estudiantes a reflexionar sobre la propia espiritualidad y religiosidad, a estudiar el hecho religioso, a analizar las tradiciones religiosas de cada uno y conocer diversas manifestaciones espirituales y religiosas presentes en la región. Cabe mencionar que también se integra a quienes se declaran ateos o agnósticos a quienes se les pide también dar razón de su manera de ver y entender la vida.

En relación a la metodología, se propician instancias de aprendizaje dialógico, esto es pasar de la 'dialéctica' a la 'dialógica' en la construcción de conocimiento, porque sólo a través del diálogo se puede encontrar la mejor forma de vivir la diversidad espiritual y religiosa en base a tolerancia, aceptación y respeto mutuo. Esto comporta un desafío especial para el profesor, quien debe entender su docencia como "comunicación bidireccional entre personas que sostienen puntos de vista significativamente diferentes sobre un tema, con el propósito de aprender más verdad sobre ese tema en la interacción con el otro,"13 lo que supone un docente abierto a aprender de sus estudiantes.

El curso se ofrece desde una orientación católica, entendida como universalidad y, por tanto, en apertura hacia las distintas tradiciones religiosas que contribuyen a responder las incógnitas de los hombres y mujeres de hoy, mediante el diálogo y el encuentro con el cristianismo en sus diversas manifestaciones y denominaciones, y con las religiones no cristianas presentes en nuestra región, con una preocupación particular por el pueblo Mapuche.

\subsection{Experiencias significativas}

${ }^{13}$ Leonard Swidler, "A dialogue on dialogue" en Death or dialogue: From the age of monologue to the age of dialogue, editado por Leonard Swidler y John B. Cobb, Londres: SCM, 1990; pp. 5678. 
El curso desarrolla lo que hemos llamado experiencias significativas. Son cuatro que se describen muy brevemente a continuación:

\section{a) Identidad religiosa}

Ya se ha dicho que el diálogo interreligioso se realiza sobre cuestiones de fe o religión sustentado en las convicciones personales profundas que movilizan existencialmente a cada persona de fe y, a pesar de que no todos los estudiantes que toman el curso son creyentes en Dios o adhieren alguna religión, es fundamental que se enfrenten al desafío de revisar su historia personal y descubrir qué personas, experiencias, y/o procesos vitales les han llevado a creer o no creer religiosamente. Este proceso de reconocimiento, esta reflexión, es el punto de partida y preparación para el diálogo y enfrenta a cada estudiante con su propia historia y verdad. Es una forma particular de indagación narrativa autobiográfica.

b) Las religiones ante la realidad contemporánea

La sociedad contemporánea se reconfigura en procesos que van dejando atrás los valores que durante mucho tiempo sirvieron para darle solidez a la estructura social. ${ }^{14}$ Actualmente la sociedad se ve enfrentada a cambios radicales, tanto a nivel social, político, económico, moral, valórico y religioso. Dichos cambios y otros, son una instancia favorable para poner en práctica actitudes de diálogo que sirvan para abrir espacios comunes. Es por ello que temas como la pobreza, el aborto, el matrimonio igualitario, la manipulación genética, el conflicto entre el Estado chileno y el Pueblo Mapuche, entre otros, se analizan en el curso, teniendo como base lo que la religión de cada participante enseña sobre esas realidades. Esta experiencia, que se desarrolla en grupos de trabajo que escogen el tema a tratar, puede ser entendida como un puente entre el diálogo intrarreligioso y el diálogo interreligioso, ya que los estudiantes tienen la oportunidad de aprender y presentar sus convicciones frente a los acontecimientos de la realidad y además escuchar y

\footnotetext{
${ }^{14}$ Zygmunt Bauman, Modernidad líquida, Ciudad de México: Fondo de Cultura Económica, 2002.
} 
aprender de los otros. Acá se descubren más aspectos comunes que diferentes, sobre todo en lo relativo a temáticas relacionadas con la defensa y dignidad de la vida humana. También hay quienes se reconocen en desacuerdo con la enseñanza de su religión o que asumen posiciones valóricas distintas, lo que los lleva a un nuevo discernimiento y a una re significación de su 'opción creyente'. ${ }^{15}$

c) Testimonio de fe

Una de las actividades fundamentales del curso es invitar y compartir con fieles de distintas religiones, quienes comparten un testimonio y comentan aspectos principales de su fe. Los estudiantes se enfrentan a una instancia de Diálogo Interreligioso. Han visitado el curso representantes de la Comunidad Islámica, la Comunidad Baháí, la Comunidad Israelita, la Iglesia Católica, diferentes iglesias Evangélicas, y de la Religión Mapuche.

d) Salir de sí al encuentro del otro

La cuarta y última experiencia es particularmente significativa porque implica una salida a terreno. Lo que se pretende es que los estudiantes dialoguen con autoridades religiosas del mundo mapuche y su entorno, familiar, natural y espiritual. Los estudiantes son recibidos por un machi en su ruka, luego de convenir previamente con él, el día y el lugar de la visita. Esto que parece ser un aspecto básico de organización, resulta ya una instancia de diálogo y apertura importante, puesto que desde la cosmovisión mapuche es fundamental que el machi se disponga espiritualmente para el encuentro, lo que implica consultar a sus mayores y observar e interpretar los signos de la naturaleza y el entorno. Los alumnos entran en la lógica de la comunión con las personas y la naturaleza (el mapu) y además de aprender sobre la cosmovisión y religión mapuche, comprenden que hay otras formas de organización y de toma de decisiones. Es, como indica el título de este

15 Antonio Bentué, La Opción Creyente, Santiago: Ediciones San Pablo, 2014. 
apartado, un salir de sí, para ir al encuentro de otro y ponerse a disposición mutuamente.

\subsection{Algunos resultados}

Uno de los resultados más significativos de este curso es el fortalecimiento de la identidad religiosa de los estudiantes. Esto sucede por varios motivos. En el diálogo sobre temas de interés y actualidad, desde distintos puntos de vista, descubren y aprecian el aporte de la cosmovisión religiosa en la cotidianeidad de la vida. Los mismos estudiantes han valorado que el encuentro y conocimiento del otro, tanto los compañeros como los testigos de otras religiones, y sobre todo su manera de pensar y creer, ha contribuido al conocimiento de sí mismos, al cuestionamiento de sus propias creencias, a reencontrarse con su historia personal y familiar. En efecto, se redescubren como parte de un contexto, una comunidad que da sentido, otorga claves de interpretación, permite mirar el mundo con unos lentes compartidos (centros de valor y poder, les llama Fowler), los cuales eventualmente pueden cambiarse por otros, o ajustarse a las propias experiencias personales.

El curso, sustentando en la perspectiva del diálogo interreligioso, ha permitido a los estudiantes conocer experiencias de fe de otras culturas, así como darse cuenta de la influencia que tienen los medios de comunicación en la conformación del imaginario que se tiene de las otras culturas y religiones. Esa situación ha ayudado a que los estudiantes se cuestionen la propia realidad local y como es presentada por los medios, estableciendo un cierto paralelismo específicamente, entre el Islam y el Pueblo Mapuche. Ambos suelen ser presentados en la prensa y en las redes con rótulos como 'terroristas' o 'violentistas', en suma, peligrosos, haciendo generalizaciones que distorsionan la imagen que la sociedad se va formando de dichas formas de vivir y creer y de cómo se relacionan con la sociedad. El conocer personalmente el testimonio de fieles musulmanes les permite romper las etiquetas y transponer a la realidad de La Araucanía el daño que provocan los prejuicios culturales y religiosos. 
Los resultados del curso, de acuerdo a la percepción recogida de los estudiantes, son hasta ahora auspiciosos. A la vez, plantean al menos dos desafíos futuros. Por una parte, la perspectiva de diálogo interreligioso aquí comentada debiera expandirse e implementarse en otras asignaturas de la oferta de formación humanista cristiana de la UC Temuco. ¿Es posible asumirla como metodología de trabajo en otros cursos? Un segundo desafío es la indagación sistemática del impacto que este tipo de curso tiene en la formación ciudadana de los estudiantes. ¿Cuánto y en qué contribuyen efectivamente? ¿Cómo se vincula este enfoque interreligioso a la convivencia sociocultural en La Araucanía?

\section{Conclusiones}

El conflicto entre el Estado de Chile y el Pueblo Mapuche, que se vive con mayor intensidad en La Araucanía, tiene un peligroso potencial de crecimiento si se mantienen las lógicas de dominación y negación del otro diferente. En el mundo entero crecen con fuerza las identidades locales, y la clave de la resolución de conflictos en los países que han avanzado en ello, ha sido el reconocimiento de la diversidad mediante mecanismos de diálogo intercultural. La Universidad Católica de Temuco, fiel a su historia y a su misión de compromiso público y regional, intenta colaborar en esa perspectiva dialógica. Lo hace, entre otras cosas, asumiendo que el diálogo interreligioso es la clave más adecuada, en el contexto universitario, para educar en la fe de sus estudiantes. Una comprensión más amplia del fenómeno religioso, en la que se observa la realidad desde el prisma de otros compañeros en el diálogo, sin renunciar a las propias convicciones si no ampliándolas, favorece la convivencia ciudadana y la búsqueda de alternativas que destraben el conflicto.

Uno de los mecanismos de la UC Temuco en esta línea es el curso electivo Manifestaciones de la espiritualidad en la Araucanía. Una mirada a la diversidad, que se ha presentado. Se trata de una experiencia de diálogo que no responde sólo a la convicción que en la formación de los estudiantes universitarios es fundamental conocer y reflexionar sobre el hecho religioso. El curso está planteado como una instancia en la que se busca que cada 
estudiante se reconozca a sí mismo en el conocimiento del otro, y desde sí mismo pueda contribuir a la construcción de una sociedad mejor, especialmente en la región de la Araucanía. Desde esta perspectiva, la idea fundamental que se ha trabajado consiste en que las personas que profesan una religión están llamadas a salir de la comodidad de sus comunidades para involucrarse en la vida social con personas que no tienen la misma cosmovisión y dar a conocer su identidad religiosa. Y quienes no adhieren a una tradición religiosa están llamados a valorar el aporte que estas hacen a la vida de los creyentes y a la paz social, cuando son entendidas desde su dimensión existencial y no sólo como mera pertenencia institucional. Hasta aquí ha resultado ser un primer paso en el que, pese a la crisis que actualmente vive la religión y las instituciones religiosas, se aprecia el ámbito religioso y espiritual. También ha sido la oportunidad para ampliar el horizonte y el campo de acción en el intento de construir una sociedad común, donde los estudiantes buscan desarrollarse considerando la dimensión religiosa como un aspecto fundamental de su ser personas y profesionales. 


\section{Bibliografía}

Artacho López, Rafael. (2009). Enseñar competencias sobre la religión. DDB. Bilbao.

Bauman, Z. (2002). Modernidad líquida. Fondo de Cultura Económica. Ciudad de México.

Bentué, A. (2014). La opción creyente. Ediciones San Pablo. Santiago, Chile.

Casaldáliga, P. y Vigil, J.M. (1992). Espiritualidad de la Liberación. Sal Terrae. Santander.

Comisión Teológica Internacional. (2012). El cristianismo y las religiones. Ediciones San Pablo. Bogotá.

Conciliar Nostra Aetate. En Concilio vaticano II. (1966). BAC. Madrid.

Dirección General de Docencia. (2016). Cuaderno No3 Competencias genéricas para la formación de profesionales integrales. Ediciones Universidad Católica de Temuco. Temuco.

Fowler, J. (1976). Stages of Faith. The Psychology of Human Development and the Quest for Meaning. San Francisco: Harper \& Row.

García, J. (2006). "Retos del Diálogo Interreligioso al Diálogo Ecuménico" en Cultura para la Esperanza 63. 
Menchaca L., A. (1959). Decreto $N^{\circ} 375$. Diócesis San José de Temuco.

Pairican, F. Malon. (2016) La rebelión del movimiento mapuche 1990-2013. Pehuén. Santiago.

Papa Francisco. (2013). Discurso al mundo universitario en la Pontificia Facultad Teológica Regional en Cerdeña (22 de septiembre de 2013).

Parks, S. (2000). Big Questions, Worthy Dreams: mentoring Young adults in their search for meaning, purpose, and faith. Jossey-Bass Inc. San Francisco.

Swidler, L. (1990). "A dialogue on dialogue" en Death or dialogue: From the age of monologue to the age of dialogue, editado por Leonard Swidler y John B. Cobb, 56-78. Londres: SCM.

Universidad Católica de Temuco. (2010). Plan de Desarrollo Institucional 2010-2020. Temuco.

Vaticano II. (1999). Declaración Nostra Aetate. Sobre las relaciones de la Iglesia con las religiones no cristianas. 